\title{
Methods
}

Fangzhou Liu, Zengjie Zhang* and Martin Buss

\section{Optimal filtering and control of network information epidemics}

\author{
Optimale Filterung und Regelung von Netzwerk-Informationsepidemien
}

https://doi.org/10.1515/auto-2020-0096

Received June 11, 2020; accepted January 8, 2021

\begin{abstract}
In this article, we propose an optimal control scheme for information epidemics with stochastic uncertainties aiming at maximizing information diffusion and minimizing the control consumption. The information epidemic dynamics is represented by a network SusceptibleInfected-Susceptible (SIS) model contaminated by both process and observation noises to describe a perturbed disease-like information diffusion process. To reconstruct the contaminated system states, we design an optimal filter which ensures minimized estimation errors in a quadratic sense. The state estimation is then utilized to develop the optimal controller, for which the optimality of the closed-loop system is guaranteed by a separation principle. The designed optimal filter and controller, together with the separation principle, form a complete solution for the optimal control of network information epidemics with stochastic perturbations. Such optimalfiltering-based control strategy is also generalizable to a wider range of networked nonlinear systems. In the numerical experiments on real network data, the effectiveness of the proposed optimal control is validated and confirmed.
\end{abstract}

Keywords: optimal filtering, optimal control, information epidemics

Zusammenfassung: In diesem Artikel schlagen wir ein optimales Regelungsschema für Informationsepidemien mit stochastischen Unsicherheiten vor, das darauf abzielt, die Informationsdiffusion zu maximieren und den Regelungsverbrauch zu minimieren. Die Dynamik der Informationsepidemie wird durch ein Netzwerk Susceptible-Infected-

\footnotetext{
*Corresponding author: Zengjie Zhang, Chair of Automatic Control Engineering (LSR), Department of Electrical and Computer Engineering, Technical University of Munich, Theresienstr. 90, 80333, Munich, Germany, e-mail: zengjie.zhang@tum.de Fangzhou Liu, Martin Buss, Chair of Automatic Control Engineering (LSR), Department of Electrical and Computer Engineering, Technical University of Munich, Theresienstr. 90, 80333, Munich, Germany, e-mails: fangzhou.liu@tum.de,mb@tum.de
}

Susceptible (SIS)-Modell dargestellt, das sowohl durch Prozess- als auch durch Beobachtungsrauschen kontaminiert ist, um einen gestörten krankheitsähnlichen Informationsdiffusionsprozess zu beschreiben. Um die kontaminierten Systemzustände zu rekonstruieren, entwerfen wir einen optimalen Filter, der minimale Schätzfehler in einem quadratischen Sinn gewährleistet. Die Zustandsschätzung wird dann verwendet, um den optimalen Regler zu entwickeln, für den die Optimalität des geschlossenen Regelkreises durch ein Separationsprinzip garantiert wird. Der entworfene optimale Filter und Regler bilden zusammen mit dem Separationsprinzip eine vollständige Lösung für die optimale Regelung von NetzwerkInformationsepidemien mit stochastischen Störgrößen. Eine solche auf optimaler Filterung basierende Regelungsstrategie ist auch auf ein breiteres Spektrum von vernetzten nichtlinearen Systemen verallgemeinerbar. In den numerischen Experimenten an realen Netzwerkdaten wird die Wirksamkeit der vorgeschlagenen optimalen Regelung validiert und bestätigt.

Schlagwörter: Optimalfilter, optimale Regelung, Informationsepidemie

\section{Introduction}

Information epidemics describe the diffusion of pathogenlike information in population [1] and play a vital role in various scenarios, e. g., campaigning, viral marketing, and rumor spreading [2-4]. The relevant research topics, such as the modeling, analysis, and control of information epidemics, have attracted wide attention in the fields of information science, social networks, and group psychology [5].

The information epidemic models, used to depict the evolvement of epidemics, are generally recognized as compartmental models that categorize the population into different compartments, e. g., susceptible (S) and infected (I) [6]. In the context of information diffusion, 
these compartments may respectively refer to unawareness and awareness of the information, or people's willingness to refuse or adopt the information. A representative and widely-studied epidemic model is the SusceptibleInfected-Susceptible (SIS) model which depicts the dynamics of the population in terms of the bidirectional transitions between compartments $\mathrm{S}$ and I. In addition, the networked information epidemic models with heterogeneous transition rates $[7,8]$ have recently gained more value than the earlier homogeneous-structure-based scalar models [9], due to the advantage of better depicting the structural and behavioral heterogeneity of social networks. Here, by homogeneous we mean that the population is well-mixed, i.e., the individuals in the network have the same chances to interact with others, while by heterogeneous we allow various individual properties. Apparently, the heterogeneous models are more flexible to be generalized to solve practical problems than the homogeneous ones. Therefore, the networked SIS model has been widely applied to popular research topics including innovation diffusion, epidemic spreading, and new technology adoption [10,11]. To this end, we focus on the networked SIS model with heterogeneous transition rates as a representative information epidemic model.

As a fundamental research issue, control of information epidemics has obtained increasingly more concerns in recent years [12]. However, with the particular interests of the previous work on the noise-free models, there have been very few results on the control of noisy epidemics, although noise is ubiquitous in practical information diffusion processes [13]. Here, noise is a generic notion to depict both the exogenous stochastic uncertainties and the inherent random properties of the epidemic models, such as the information loss through the network (due to imperfect conveyance) and the understanding discrepancies among the individuals (due to various languages, culture, gender, or education background). When these factors affect the information epidemics, we formulate them as stochastic processes in the model and refer to the former as the process noise and the latter as the observation noise. Due to the involvement of the stochastic noise, the conventional deterministic control schemes cannot be applied, since explicit state-feedback is not available. Instead, the design of the controller should be based on the precise reconstruction of system states. Conventionally, the modeling noise in innovation diffusion is introduced as white noise with Gaussian properties [9], which motivates us to seek for an optimal controller for noisy epidemics based on optimal state filtering. Nevertheless, both the inherent nonlinearity of information epidemics and the strong coupling in the network render great challenges to such a control scheme which are still not overcome by the conventional filteringand-control solutions [14-19]. Filling this gap is the major focus of our work.

The main contribution of this article is, for the first time, to develop an optimal-filtering-based optimal control scheme for network information epidemic models, considering both the process and observation noises. As a representative information epidemics model, the networked SIS model with heterogeneous transition rates is taken into consideration. Specifically, the optimal filtering is concerned with the minimum quadratic estimation errors, which ensures precise estimation with respect to the second-order stochastic moments of the system states, and the optimal control aims at maximizing the information diffusion taking into account the input consumption. The optimality of both the state estimation and feedback control is guaranteed by a separation principle. Based on this, we are able to solve a series of problems that are concerned with the stochastic properties of information epidemics, such as innovation diffusion, rumor mitigation, and viral marketing with unmodeled uncertainties. From a general perspective, the optimal filtering and control developed in this article can be extended to a wide range of stochastic nonlinear network systems, such as the smart grid system affected by random environmental factors.

The remainder of this article is organized as follows: In Section 2, the network SIS epidemic model with process noise and observation noise is introduced. We then formulate the problem to be solved in this article. The optimal filter and the optimal control law are respectively presented in Section 3 and Section 4. Numerical experiments are conducted in Section 5 to illustrate the effectiveness of the proposed results. Finally, we conclude this article in Section 6.

\section{Notations}

Let $\mathbb{R}, \mathbb{R}_{\geq 0}$, and $\mathbb{R}^{n}$ respectively denote the set of real numbers, non-negative real numbers, and $n$-dimensional vectors. 1(0) and $I$ represent the all-ones (zero) vector and the identity matrix with proper dimension, respectively. $\mathrm{E}(\cdot)$ denotes the expectation of certain event and $\mathrm{E}(\cdot \cdot \cdot)$ refers to the conditional expectation. For a vector $\xi \operatorname{diag}(\xi)$ stands for a diagonal matrix whose $i$-th diagonal entry equals the $i$-th component of $\xi$. $(\mathcal{S}, \mathcal{F}, \mathcal{P})$ denotes a probability space, where $\mathcal{S}$ is the sampling space, $\mathcal{F}$ is the $\sigma$-algebra defined on $\mathcal{S}$, and $\mathcal{P}$ is the probability measure. o represents the Hadamard product, i. e., the element-wise product of two matrices, and for arbitrary matrices $K$ and $L$ with the same dimensions, we have

$$
K \circ L=L \circ K,(K \circ L)^{\top}=K^{\top} \circ L^{\top}=L^{\top} \circ K^{\top} .
$$




\section{Problem formulation}

Consider an $n$-node strongly connected weighted directed graph $\mathcal{G}=(\mathcal{V}, \mathcal{E}, A)$ where $\mathcal{V}=\{1,2, \ldots, n\}$ and $\mathcal{E} \subseteq \mathcal{V} \times \mathcal{V}$ are the set of labeled vertices and the set of edges, respectively. $A=\left[a_{i j}\right]$ is the nonnegative adjacency matrix where a nonzero entry $a_{i j}$ corresponds to an edge $(j, i)$, i. e., node $i$ can influence node $j$. The continuous-time network SIS model on the graph $\mathcal{G}$ reads

$$
\dot{x}_{i}(t)=\left(1-x_{i}(t)\right) \sum_{j=1}^{n} \beta_{j}(t) a_{i j} x_{j}(t)-\gamma_{i}(t) x_{i}(t), t \in \mathbb{R}_{\geq 0},
$$

for all $i \in \mathcal{V}$, where $x_{i}(t) \in \mathbb{R}$ is the infection probability of node $i$. Another interpretation of $x_{i}$, from the perspective of meta-population, is the infection proportion of $i$-th group (e.g, a city or a state). $\beta_{i}(t), \gamma_{i}(t) \in \mathbb{R}$ are respectively the time-varying infection rate and curing rate of node $i$ at time instant $t$. In accordance with previous literature, the infection process is considered to be proactive while the curing process is passive $[8,20]$. In this article, we consider both process noise and observation noise in the information epidemics. From this perspective, the SIS model with stochastic noise is formulated as

$$
\mathrm{d} x_{i}=\left(\left(1-x_{i}\right) \sum_{j=1}^{n} a_{i j} \beta_{j}(t) x_{j}-\gamma_{i}(t) x_{i}\right) \mathrm{d} t+e_{i}(t) \mathrm{d} \omega_{i},
$$

where $e_{i}(t) \in \mathbb{R}$ is a scalar coefficient and $\omega_{i}(t) \in \mathbb{R}$ is a standard Wiener process defined on a probability space $(\mathcal{S}, \mathcal{F}, \mathcal{P})$, such that $\mathrm{E}\left(\omega_{i}\right)=0$ and $\mathrm{E}\left(\omega_{i}^{2}\right)=t$. The compact form of (2) reads

$$
\mathrm{d} x_{t}=\left(\left(I-X_{t}\right) A X_{t} \beta_{t}-X_{t} y_{t}\right) \mathrm{d} t+E_{t} \mathrm{~d} \omega_{t},
$$

where $x_{t}=\left[x_{1}, x_{2}, \ldots, x_{n}\right]^{\top}, \beta_{t}=\left[\beta_{1}, \beta_{2}, \ldots, \beta_{n}\right]^{\top}, \gamma_{t}=$ $\left[\gamma_{1}, \gamma_{2}, \ldots, \gamma_{n}\right]^{\top}, \omega_{t}=\left[\omega_{1}, \omega_{2}, \ldots, \omega_{n}\right]^{\top}, X_{t}=\operatorname{diag}\left(x_{t}\right)$, and $E_{t}=\operatorname{diag}\left(\left[e_{1}, e_{2}, \ldots, e_{n}\right]\right)$. Note that we use $x_{t}$ to represent the state vector and define $x_{i}$ as its $i$-th element. Ambiguity is avoided since $t \in \mathbb{R}_{\geq 0}$ and $i \in \mathcal{V}$ belong to different domains. The system state $x_{t}$ is not directly measurable, but hidden by the following observation process

$$
\mathrm{d} y_{t}=H_{t} x_{t} \mathrm{~d} t+F_{t} \mathrm{~d} v_{t}
$$

where $H_{t}, F_{t} \in \mathbb{R}^{n \times n}$ are time-dependent matrices, and $y_{t} \in \mathbb{R}^{n}, y_{t}=\mathcal{Y}_{t}$ is the result of an observation, and $v_{t}=\left[v_{1}, v_{2}, \ldots, v_{n}\right]^{\top} \in \mathbb{R}^{n}$ is a $n$-dimensional stochastic process. For each $i=1,2, \cdots, n, v_{i}$ is a standard Wiener process defined on $(\mathcal{S}, \mathcal{F}, \mathcal{P})$, and $\mathrm{E}\left(v_{i}\right)=0, \mathrm{E}\left(v_{i}^{2}\right)=t$. Therefore, $\mathrm{d} \omega_{t}$ and $\mathrm{d} v_{t}$ denote Gaussian white noise processes whose covariance matrices are respectively constant matrices $\Omega \delta(t)$ and $V \delta(t)$, where $\Omega, V \in \mathbb{R}^{n \times n}$ are constants assumed to be known a-priori and $\delta(t) \in \mathbb{R}$ is the Dirac delta function.
Remark 1. The dynamics (3) are an Itô's-formula-based stochastic model with n-dimensional Gaussian white noise $\mathrm{d} \omega_{t}$ and $\mathrm{d} v_{t}$. In the context of information epidemics, $\mathrm{d} w_{t}$ and $\mathrm{d} v_{t}$ respectively denote the process noise and the observation noise. By setting $E_{t}$ and $F_{t}$ as diagonal matrices, we assume that each noise channel of model (3) is mutually independent. Nevertheless, it is worth noting that our approach developed in this article is not limited to this assumption but also apply to arbitrary $E_{t}$ and $F_{t}$.

In this article, we are targeting to solving following problem.

Problem 1. For the information epidemics model (3) on a weighted strongly connected digraph $\mathcal{G}=\{\mathcal{V}, \mathcal{E}, A\}$ and its observation process in (4), we solve the following two stages of problems:

(1) Optimal filtering: reconstruct the estimation $\widehat{x}_{t}$ using the observation $\mathcal{Y}_{t}$, such that the following estimation criterion is minimized,

$$
J_{o}=\mathrm{E}\left(\left(x_{t}-\widehat{x}_{t}\right)^{\top}\left(x_{t}-\widehat{x}_{t}\right) \mid \mathcal{Y}_{t}\right) .
$$

(2) Optimal control: design a control law for the infection rate of dynamics (3), $\beta_{t}=u_{t}\left(\widehat{x}_{t}\right)$, such that the following control criterion is minimized.

$$
J_{c}=\mathrm{E}\left[\int_{t_{0}}^{t_{f}}\left(-p_{t}^{\top} x_{t}+\frac{1}{2} \beta_{t}^{\top} R_{t} \beta_{t}\right) \mathrm{d} t-\psi^{\top} x_{t_{f}}\right],
$$

where $p_{t} \in \mathbb{R}^{n}$ is an element-wisely positive function vector, $R_{t} \in \mathbb{R}^{n \times n}$ is a positive-definite time-variant matrix, $\psi \in \mathbb{R}^{n}$ is a constant vector, and $x_{t_{f}} \in \mathbb{R}^{n}$ is the system state at the terminal instant $t_{f}$.

Remark 2. The filtering cost in (5) is an essential criterion of the conventional optimal filtering paradigm [21] which takes the quadratic form of the state-estimation error. Meanwhile, in the control cost (6), $p_{t}^{\top} x_{t}$ and $\psi^{\top} x_{t_{f}}$ stand for the weighted reward expectations which attempt to maximize the information diffusion, and the quadratic term of $\beta_{t}$ denotes the intention to minimize the consumption of the control efforts [12, 22]. The successive design-procedure of the filter and the controller in Problem 1 is justified by a separation principle which is to be interpreted in Sec. 4.

Remark 3. By designing a control scheme for the infection rate $\beta_{t}$, we suppose that the network achieves the desired performance through a word-of-mouth mechanism, i. e., agents are trying to influence their social neighbors via the communication topology [7, 23]. Therefore, Problem 1 clearly describes a scenario in which we accelerate the diffusion of the information through the network by influencing 
the infection rates with the concern of control consumption. Note that how to implement the control is task-specific. For example, in a recommendation system or advertising, the frequency of the "information push" is a direct input. For more general settings, our approach is also applicable for controlling $y_{t}$ or both the transition rates in (3) by straightforward extension.

\section{Optimal filtering}

In this section, we design the optimal filter for the network information epidemic model in (3). For brevity, we represent the system dynamics (3) as

$$
\mathrm{d} x_{t}=f_{t} \mathrm{~d} t+E_{t} \mathrm{~d} \omega_{t}
$$

where $f_{t}=\left(I-X_{t}\right) A X_{t} \beta_{t}-X_{t} y_{t}$ is the deterministic nonlinear process function. It is known from [21] that the optimal estimation $\widehat{x}_{t}$ of the system state $x_{t}$ with observation $\mathcal{Y}_{t}$, with respect to the estimation criterion (5) is

$$
\widehat{x}_{t}=\mathrm{E}\left(x_{t} \mid \mathcal{Y}_{t}\right)
$$

with an estimation variance $P_{t}=\mathrm{E}\left(\left(x_{t}-\widehat{x}_{t}\right)\left(x_{t}-\widehat{x}_{t}\right)^{\top} \mid \mathcal{Y}_{t}\right)$, and (8) renders an unbiased estimation, i. e., $\mathrm{E}\left(\widehat{x}_{t}\right)=\mathrm{E}\left(x_{t}\right)$. In the follows, we continue to use the notation $\hat{r}=\mathrm{E}\left(\cdot \mid \mathcal{Y}_{t}\right)$ as (8) to represent the conditional expectations. According to [21], the optimal estimation $\widehat{x}_{t}$ is formulated as follows

$$
\mathrm{d} \widehat{x}_{t}=\widehat{f}_{t} \mathrm{~d} t+P_{t} H_{t}^{\top}\left(F_{t} V F_{t}^{\top}\right)^{-1}\left(\mathrm{~d} \mathcal{Y}_{t}-H \widehat{x}_{t} \mathrm{~d} t\right)
$$

with the estimation variance

$$
\begin{aligned}
\mathrm{d} P_{i j}= & \left(\widehat{x_{i} f_{j}}-\widehat{x}_{i} \widehat{f}_{j}\right)+\left(\widehat{f_{i} x_{j}}-\widehat{f}_{i} \widehat{x}_{j}\right)+\left(E_{t} \Omega E_{t}^{\top}\right)_{i j} \\
& -\sum_{k=1}^{n} \sum_{r=1}^{n}\left(\widehat{x_{i} x_{k}}-\widehat{x}_{i} \widehat{x}_{k}\right)\left(H_{t}^{\top}\left(F_{t} V F_{t}^{\top}\right)^{-1} H_{t}\right)_{k r} \\
& \left.\times\left(\widehat{x_{r} x_{j}}-\widehat{x}_{r} \widehat{x}_{j}\right)\right) \mathrm{d} t \\
+ & \sum_{k=1}^{n}\left(\widehat{x_{i} x_{j} x_{k}}-\widehat{x_{i} x_{j} \widehat{x}_{k}}-\widehat{x_{k} x_{j} \widehat{x}_{i}}-\widehat{x_{i} x_{k} \widehat{x}_{j}}\right. \\
& \left.+2 \widehat{x_{i} \widehat{x}_{j} \widehat{x}_{k}}\right)\left(H^{\top}\left(F_{t} V F_{t}^{\top}\right)^{-1}\left(\mathrm{~d} y_{t}-H \widehat{x}_{t} \mathrm{~d} t\right)\right)_{i j},
\end{aligned}
$$

where $(\cdot)_{i}$ is the $i$-th element of a vector and $(\cdot)_{i j}$ is the element of a matrix at the $i$-th row and $j$-th column. For the estimation $\widehat{x}_{t}$, we propose the following assumption.

Assumption 1. [24] All third-order central moments of the system state $x_{t}$ are zero, i.e., $\forall i, j, k=1,2, \cdots, n$,

$$
\mathrm{E}\left(\left(x_{i}-\widehat{x}_{i}\right)\left(x_{j}-\widehat{x}_{j}\right)\left(x_{k}-\widehat{x}_{k}\right) \mid \mathcal{Y}_{t}\right)=0 .
$$

Based on Assumption 1, the following theorem guarantees the optimality of the proposed optimal filter.

Theorem 1. A feasible optimal filter solution for Problem 1-(1), following the paradigm (9) and (10), is formulated as

$$
\begin{gathered}
\mathrm{d} \widehat{x}_{t}=\left(\left(I-\widehat{X}_{t}\right) A B_{t} \widehat{x}_{t}-\Gamma_{t} \widehat{x}_{t}-A \circ P_{t} \beta_{t}\right) \mathrm{d} t \\
+P_{t} H_{t}^{\top}\left(F_{t} V F_{t}^{\top}\right)^{-1}\left(\mathrm{~d} \mathcal{Y}_{t}-H_{t} \widehat{x}_{t} \mathrm{~d} t\right), \\
\dot{P}_{t}=\left(I-\widehat{X}_{t}\right) A B_{t} P_{t}+\Gamma_{t} P_{t}+P_{t} B_{t}^{\top} A^{\top}\left(I-\widehat{X}_{t}\right)+P_{t} \Gamma_{t} \\
-\operatorname{diag}\left(A B_{t} \widehat{x}_{t}\right) P_{t}-P_{t} \operatorname{diag}\left(A B_{t} \widehat{x}_{t}\right) \\
+E_{t} \Omega E_{t}^{\top}-P_{t} H_{t}^{\top}\left(F_{t} V F_{t}^{\top}\right)^{-1} H_{t} P_{t},
\end{gathered}
$$

where $\widehat{X}_{t}=\operatorname{diag}\left(\widehat{x}_{t}\right), B_{t}=\operatorname{diag}\left(\beta_{t}\right), \Gamma_{t}=\operatorname{diag}\left(y_{t}\right)$.

Proof. See Appendix for detailed proof.

Remark 4. Compared to the conventional optimal filter for linear systems, also known as the Kalman filter [25], the proposed optimal filter (12) for the information epidemic model (3) shows distinguished features, i.e., the additional terms $A \circ P_{t} \beta_{t}$ in (12a) and diag $\left(A B_{t} \widehat{x}_{t}\right) P_{t}$ in (12b). These extra terms are brought up by the nonlinearity and inherent coupling of the information epidemic models. Assumption 1 is based on the property of Gaussian random variables that all the odd conditional moments of the estimation errors $x_{t}-\hat{x}_{t}$ are zero [24]. As a result, the filter in (12) ensures a precise state estimation with respect to the second-order moments of $x_{t}-\widehat{x}_{t}$, which is generally higher than the conventional Extended Kalman filter [26] and comparable with an unscented Kalman filter [27].

\section{Optimal controller design}

Now, let us investigate the optimal control solution for Problem 1-(2). It is known that, for linear systems, the filter and controller can be designed independently while guaranteeing the stability of the closed-loop dynamics, which is referred to as the separation principle. For nonlinear systems, however, only a few types of them ensure similar properties [28]. To justify the feasibility of the independent design of the filter and the controller in this article, it is necessary to investigate the existence of the separation principle for the network SIS model (3) with the corresponding optimal criterion (6).

It is known that the optimal state reconstruction (12) renders an unbiased estimation, i.e., $\mathrm{E}\left(\widehat{x}_{t}\right)=\mathrm{E}\left(x_{t}\right)$, from which we have

$$
J_{c}=\int_{t_{0}}^{t_{f}}\left(-p_{t}^{\top} \mathrm{E}\left(x_{t}\right)+\frac{1}{2} \beta_{t}^{\top} R_{t} \beta_{t}\right)-\psi^{\top} \mathrm{E}\left(x_{t_{f}}\right)=J_{\hat{c}},
$$


where

$$
J_{\hat{c}}=\mathrm{E}\left[\int_{t_{0}}^{t_{f}}\left(-p_{t}^{\top} \widehat{x}_{t}+\frac{1}{2} \beta_{t}^{\top} R_{t} \beta_{t}\right)-\psi^{\top} \widehat{x}_{t_{f}}\right]
$$

is the control criterion that only depends on the estimated state $\widehat{x}_{t}$ dominated by the following dynamics,

$$
\dot{\hat{x}}_{t}=\left(\left(I-\widehat{X}_{t}\right) A \widehat{X}_{t}-A \circ P_{t}\right) \beta_{t}-\widehat{X}_{t} y_{t},
$$

which is the deterministic part of (12a), since the term $P_{t} H_{t}^{\top}\left(F_{t} V F_{t}^{\top}\right)^{-1}\left(\mathrm{~d} \mathcal{Y}_{t}-H_{t} \widehat{x}_{t} \mathrm{~d} t\right)$ in (12a) is a Gaussian white noise [29]. This indicates that the optimal stochastic control law $u_{t}\left(x_{t}\right)$ for system (3) with respect to the criteria (6) is equivalent to the deterministic one $u_{t}\left(\widehat{x}_{t}\right)$ for the deterministic dynamics (14) and the cost criterion (13), which formulates the separation principle for the information epidemic models (3). Having this, we are ready to propose the optimal control solution for Problem 1-(2).

Theorem 2. A feasible optimal control solution for Problem 1-(2), minimizing the cost criterion (6), is formulated as

$$
\beta_{t}=-R_{t}^{-1}\left(P_{t}^{\circ} A^{\top}-\widehat{X}_{t} A^{\top}\left(I-\widehat{X}_{t}\right)\right) q_{t},
$$

where $q_{t} \in \mathbb{R}^{n}$ is a time-dependent vector function dominated by

$$
\begin{aligned}
\dot{q}_{t}= & \Gamma_{t} q_{t}-p_{t}+\operatorname{diag}\left(A^{\top}\left(I-\widehat{X}_{t}\right) q_{t}\right) R_{t}^{-1}\left(P_{t} \circ A^{\top}\right) q_{t} \\
& -\frac{1}{2} \operatorname{diag}\left(A^{\top}\left(I-\widehat{X}_{t}\right) q_{t}\right) R_{t}^{-1} \widehat{X}_{t} A^{\top}\left(I-\widehat{X}_{t}\right) q_{t},
\end{aligned}
$$

with terminal condition $q_{t_{f}}=\psi$.

Proof. According to the separation principle, we transform Problem 1-(2) into the optimal controller design for the deterministic dynamics (14) with the criteria (13). Therefore, a feasible optimal control law $\beta_{t}=u_{t}\left(\widehat{x}_{t}\right)$ ensures the existence of a value function $V\left(\widehat{x}_{t}, t\right)$, where $V$ : $\mathbb{R}^{n} \times \mathbb{R}^{+} \rightarrow \mathbb{R}$, such that the following Hamilton-JacobiBellman (HJB) equation holds,

$$
\begin{aligned}
-\frac{\partial V}{\partial t}= & \frac{\partial V}{\partial \widehat{x}_{t}^{\top}}\left(\left(I-\widehat{X}_{t}\right) A \widehat{X}_{t} \beta_{t}-\widehat{X}_{t} y_{t}-A \circ P_{t} \beta_{t}\right) \\
& -p_{t}^{\top} \widehat{x}_{t}+\frac{1}{2} \beta_{t}^{\top} R_{t} \beta_{t},
\end{aligned}
$$

with respect to the terminal condition.

$$
V\left(\widehat{x}_{t_{f}}, t_{f}\right)=-\psi^{\top} \widehat{x}_{t_{f}} .
$$

Therefore, we define the value function as

$$
V\left(\widehat{x}_{t}, t\right)=-q_{t}^{\top} \widehat{x}_{t}-g_{t}
$$

where $q_{t} \in \mathbb{R}^{n}$ and $g_{t} \in \mathbb{R}$ are time-variant vector and scalar respectively dominated by (15b) and the following dynamics,

$$
\dot{g}_{t}=-\frac{1}{2} q_{t}^{\top}\left(A \circ P_{t}\right) R_{t}^{-1}\left(P_{t} \circ A^{\top}\right) q_{t}
$$

By (18), we have

$$
\frac{\partial V}{\partial t}=-\dot{q}_{t}^{\top} \widehat{x}_{t}-\dot{g}_{t}, \frac{\partial V}{\partial \widehat{x}_{t}}=-q_{t} .
$$

Therefore, substituting (20) and (15a) to the HJB equation (16) leads to

$$
\begin{aligned}
& \dot{q}_{t}^{\top} \widehat{x}_{t}+\dot{g}_{t}=-\frac{1}{2} q_{t}^{\top}\left(A \circ P_{t}-\left(I-\widehat{X}_{t}\right) A \widehat{X}_{t}\right) R_{t}^{-1} \\
& \quad \times\left(P_{t}^{\circ} A^{\top}-\widehat{X}_{t} A^{\top}\left(I-\widehat{X}_{t}\right)\right) q_{t}+q_{t}^{\top} \Gamma_{t} \widehat{x}_{t}-p_{t}^{\top} \widehat{x}_{t} .
\end{aligned}
$$

It is easy to verify that the equation (21) holds by substituting (15b) and (19) to the left-hand side of (21). Therefore, the control law $\beta_{t}=u_{t}\left(\widehat{x}_{t}\right)$ in (15a) and the value function $V\left(\widehat{x}_{t}, t\right)(18)$ ensure the holding of the HJB equation (16), which indicates that the control law in (15a) is an optimal solution of Problem 1-(2).

Remark 5. Note that Theorem 2 provides a sufficient solution of Problem 1-(2). By sufficient we mean that the proposed controller (15a) might not be the unique solution, but it certainly ensures the optimality of the control criterion (13). A different optimal controller from (15a) might be found by defining another value function rather than (18), such that the HJB equation (16) holds, which, however, is beyond the scope of this article.

Until now, (12), (15a) and (15b) formulate the optimal filtering-and-control framework for the stochastic perturbed information epidemics (3) with respect to the optimality criteria (5) and (6). Note that (12) and (15b) are respectively differential equations with an initial condition and a terminal condition. Thus, we resort to the forwardbackward sweep method in [8] to solve the numerical solution governed by these equations.

\section{Simulation}

In this section, we conduct a numerical experiment to evaluate the performance of the proposed optimal filtering-and-control framework for network information epidemics. The simulation is set up on a real-world scenario, a high-school social network in Illinois which describes the friendships between the school boys [30]. We 
construct a 67-node graph by selecting the largest strongly connected component of the network and consider the information spreading through this graph, which corresponds to the model in (3). The information propagating through this network can be the latest news of a soccer star or a recent base-ball match. Therefore, corresponding to the epidemics model (3), the state of the individuals represents to what probability the boys are aware of or adopt the information. For brevity, all model parameters are determined as constant. Specifically, we set $\beta_{t}=\beta \in(0.1,0.15)^{n}$, $\gamma_{t}=\gamma \in(0.2,0.25)^{n}, E_{t}=F_{t}=H_{t}=I_{n}, t_{f}=50$, and $p_{t}=\psi=\mathbf{1}_{n}$. Note that $R_{t}$ is configured as a fixed diagonal matrix whose $i$-th diagonal entry is proportional to the number of the in-neighbors of node $i$ with coefficient 0.2. $\mathrm{d} \omega$ and $\mathrm{d} v$ are generated as Gaussian white noise with zero means and variance 0.01 . The initial condition of dynamics (3) is randomly chosen in the set $x_{t} \in\left(0, \frac{1}{3}\right)^{n}$. The evolvement of the information epidemics without control $\left(\beta_{t}=\beta\right)$ is illustrated in Fig. 1a. It is shown that, in this situation, the aware probabilities of all individuals decay to zero, which is not a satisfactory results for our intention of information diffusion.

Therefore, for this networked epidemics model, we solve the optimal filter $\hat{x}_{t}$ and the optimal controller $\beta_{t}=$ $u_{t}\left(\widehat{x}_{t}\right)$ according to the differential equations (12) and (15b). The initial condition of the estimated system (12a) is randomly chosen in $\hat{x}_{0}=\left(0, \frac{1}{3}\right)^{n}$, and $P_{0}$ is set as a positive definite matrix with $P_{i j} \in\left(0,10^{-6}\right)$ at the initial time instant $t_{0}$. The optimal filter (12) and the optimal controller (15) are solved using the forward-backward sweep method in [8]. By applying the optimal filter-and-control solution to the information epidemic model, we obtain the controlled results shown in Fig. $1 \mathrm{~b}$. As it is manifested, the proposed optimal control drives $x_{t}$ to an average infection probability around 0.6. This indicates that the aware probabilities of all the individuals are brought to a comparably high level (very likely to be aware of the information), which reflects the success of the proposed information diffusion control. The estimated system state $\widehat{x}_{t}$ and the estimation error $x_{t}-\widehat{x}_{t}$ are respectively shown in Fig. 1c and Fig. 1d. It is noticed that the estimation error converges to zero as time increases, which confirms the precision of the optimal filter. Therefore, this simulation reveals the effectiveness of the proposed optimal filter and optimal control for network information epidemics. Since the simulation is conducted on a real-world data set, the evaluation results are quite promising to serve as a reference for the practical policy design.

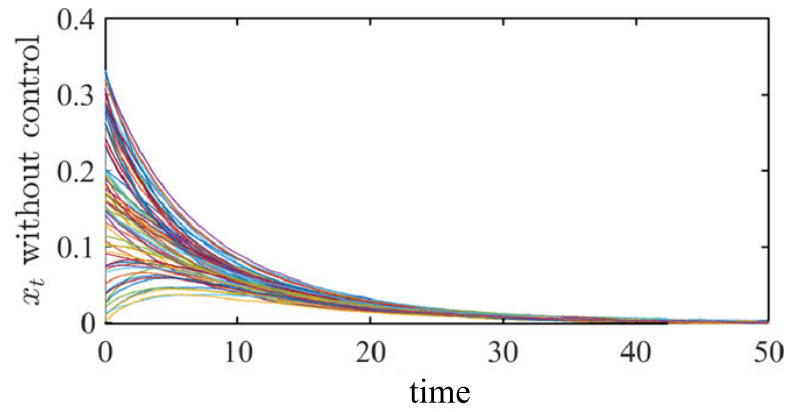

(a) $x_{t}$ without control.

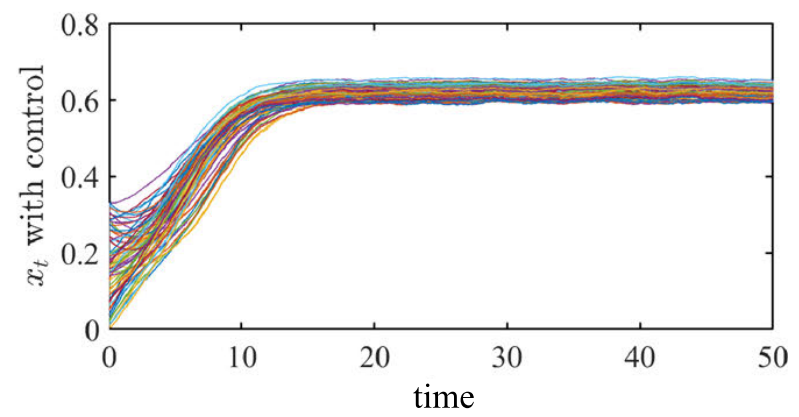

(b) $x_{t}$ with control.

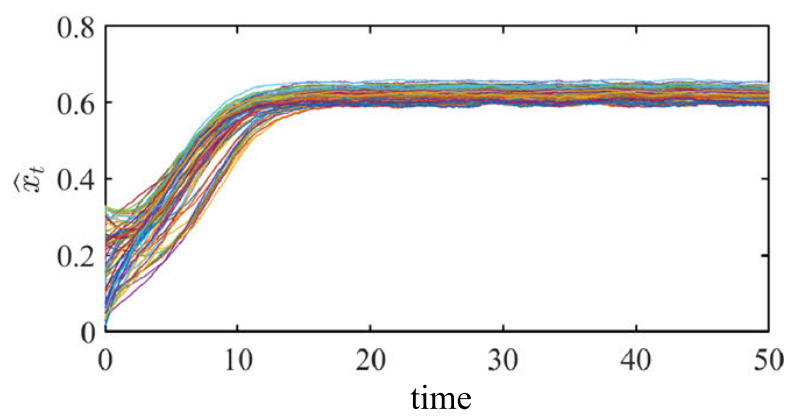

(c) $\widehat{x}_{t}$ with control.

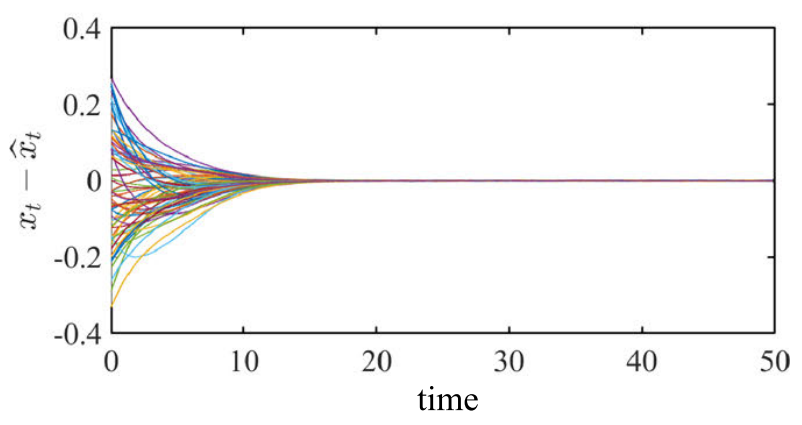

(d) Error between $x_{t}$ and $\widehat{x}_{t}$.

Figure 1: Performance of the proposed optimal filter and optimal control for network SIS model on a 67-node network. (a) and (b): By introducing the optimal control in (15a) the awareness of the information increases drastically. (c) and (d): The estimation of $x_{t}$ obtained by using the optimal filter in (12) approaches to the real value. 


\section{Conclusion}

The main focus of this paper is on solving the optimal control problem for network information epidemics with process and observation noises. The main challenge of this work lies in the handling of the nonlinearity and deep coupling brought up by the networked structure. In this paper, we overcome this challenge by independently designing an optimal filter and an optimal controller based on a proposed separation principle for the information epidemics model. By applying the proposed framework, for the first time, we are able to influence the infection rates to maximize information diffusion and minimize the effort consumption on an epidemics network, even with stochastic uncertainties on both structure and observation. From a general perspective, this framework is also promising to be applied to a wider range of network nonlinear systems that are perturbed with stochastic uncertainties, such as the networked power grid system with current perturbation. For the future work, we will be dedicated to applying the proposed methods to solving practical problems. For example, analysis and prediction of the evolution of epidemics and robust control of large-scale power grid will be our most preferred interests.

Funding: Zengjie Zhang was supported by Chinese Government Scholarship, Grant Number: 201506120029.

\section{Appendix}

Proof of Theorem 1. The main technical point of this proof is to calculate the complex conditional expectations for the estimation dynamics (9) and the variance evolvement (10). From (9), we have

$$
\begin{aligned}
\widehat{f}_{i}= & \sum_{k=1}^{n} a_{i k} \beta_{k} \widehat{x}_{k}-\gamma_{i} \widehat{x}_{i}-\sum_{k=1}^{n} a_{i k} \beta_{k} \widehat{x_{i} x_{k}} \\
= & \sum_{k=1}^{n} a_{i k} \beta_{k} \widehat{x}_{k}-\gamma_{i} \widehat{x}_{i}-\sum_{k=1}^{n} a_{i k} \beta_{k} \widehat{x}_{i} \widehat{x}_{k} \\
& -\sum_{k=1}^{n} a_{i k} \beta_{k}\left(\widehat{x_{i} x_{k}}-\widehat{x}_{i} \widehat{x}_{k}\right) \\
= & \left(1-\widehat{x}_{i}\right) \sum_{k=1}^{n} a_{i k} \beta_{k} \widehat{x}_{k}-\gamma_{i} \widehat{x}_{i}-\sum_{k=1}^{n} a_{i k} \beta_{k} P_{i k} .
\end{aligned}
$$

Therefore, for (10), we obtain

$$
\begin{aligned}
& \widehat{x_{j} f_{i}}=\sum_{k=1}^{n}\left(a_{i k} \beta_{k} \widehat{\chi_{k} x_{j}}-a_{i k} \beta_{k} \widehat{\bar{x}_{i} x_{j} x_{k}}\right)-\gamma_{i} \widehat{x_{i} x_{j}}, \\
& \widehat{x}_{j} \hat{f}_{i}=\sum_{k=1}^{n}\left(a_{i k} \beta_{k} \widehat{x}_{k} \widehat{x}_{j}-a_{i k} \beta_{k} \widehat{x_{i} x_{k} \widehat{x}_{j}}\right)-\gamma_{i} \widehat{x}_{i} \widehat{x}_{j},
\end{aligned}
$$

and

$$
\begin{aligned}
\left(\widehat{x_{j} f_{i}}-\widehat{x}_{j} \widehat{f}_{i}\right)= & \sum_{k=1}^{n} a_{i k} \beta_{k} P_{k j}-\gamma_{i} P_{i j} \\
& -\sum_{k=1}^{n} a_{i k} \beta_{k}\left(\widehat{x_{i} x_{j} x_{k}}-\widehat{x_{j}} \widehat{x_{i} x_{k}}\right) .
\end{aligned}
$$

Additionally, it is known that

$$
\begin{aligned}
& \sum_{k=1}^{n} a_{i k} \beta_{k} \widehat{x}_{k} P_{i j}=\sum_{k=1}^{n} a_{i k} \beta_{k}\left(\widehat{x_{i} x_{j} \widehat{x}_{k}}-\widehat{x}_{i} \widehat{x}_{j} \widehat{x}_{k}\right), \\
& \sum_{k=1}^{n} a_{i k} \beta_{k} \widehat{x}_{i} P_{k j}=\sum_{k=1}^{n} a_{i k} \beta_{k}\left(\widehat{x_{k} x_{j} \widehat{x}_{i}}-\widehat{x}_{k} \widehat{x}_{j} \widehat{x}_{i}\right) .
\end{aligned}
$$

Therefore, from Assumption 1 we know,

$$
\begin{aligned}
& \mathrm{E}\left(\left(x_{i}-\widehat{x}_{i}\right)\left(x_{j}-\widehat{x}_{j}\right)\left(x_{k}-\widehat{x}_{k}\right) \mid \mathcal{Y}_{t}\right) \\
& \quad=\widehat{x_{i} x_{j} x_{k}}+2 \widehat{x}_{i} \widehat{x}_{j} \widehat{x}_{k}-\widehat{x}_{i} \widehat{x_{j} x_{k}}-\widehat{x}_{j} \widehat{x_{i} x_{k}}-\widehat{x}_{k} \widehat{x_{i} x_{j}} \\
& \quad=0, \forall i, j, k=1,2, \cdots, n,
\end{aligned}
$$

which leads (24a) and (24b) to

$$
\begin{aligned}
\sum_{k=1}^{n} a_{i k} \beta_{k} \widehat{x}_{k} P_{i j} & +\sum_{k=1}^{n} a_{i k} \beta_{k} \widehat{x}_{i} P_{k j} \\
& =\sum_{k=1}^{n} a_{i k} \beta_{k}\left(\widehat{x_{i} x_{j} x_{k}}-\widehat{x}_{j} \widehat{x_{i} x_{k}}\right) .
\end{aligned}
$$

Thus, substituting (26) to (23) we obtain

$$
\begin{aligned}
\left(\widehat{x_{j} f_{i}}-\widehat{x}_{j} \widehat{f}_{i}\right)= & \left(1-\widehat{x}_{i}\right) \sum_{k=1}^{n} a_{i k} \beta_{k} P_{k j} \\
& -\left(\gamma_{i}+\sum_{k=1}^{n} a_{i k} \beta_{k} \widehat{x}_{k}\right) P_{i j} \\
\left(\widehat{x_{i} f_{j}}-\widehat{x}_{i} \widehat{f}_{j}\right)= & \left(1-\widehat{x}_{j}\right) \sum_{k=1}^{n} a_{j k} \beta_{k} P_{k i} \\
& -\left(\gamma_{j}+\sum_{k=1}^{n} a_{j k} \beta_{k} \widehat{x}_{k}\right) P_{i j} .
\end{aligned}
$$

Substituting (22) and (27) respectively to (9) and (10), we obtain the optimal filter for model (3) with the observation process in (4) as

$$
\begin{aligned}
\mathrm{d} \widehat{x}_{i}= & \left(\left(1-\widehat{x}_{i}\right) \sum_{k=1}^{n} a_{i k} \beta_{k} \widehat{x}_{k}-\gamma_{i} \widehat{x}_{i}-\sum_{k=1}^{n} a_{i k} \beta_{k} P_{i k}\right) \mathrm{d} t \\
& +\left(P_{t} H_{t}^{\top}\left(F_{t} V F_{t}^{\top}\right)^{-1}\left(\mathrm{~d} y_{t}-H \widehat{x}_{t} \mathrm{~d} t\right)\right)_{i}, \\
\dot{P}_{i j}= & \left(1-\widehat{x}_{i}\right) \sum_{k=1}^{n} a_{i k} \beta_{k} P_{k j}+\left(1-\widehat{x}_{j}\right) \sum_{k=1}^{n} a_{j k} \beta_{k} P_{k i} \\
& -\left(\gamma_{i}+\gamma_{j}+\sum_{k=1}^{n}\left(a_{i k} \beta_{k}+a_{j k} \beta_{k}\right) \widehat{x}_{k}\right) P_{i j}+\left(E_{t} \Omega E_{t}^{\top}\right)_{i j} \\
& -\sum_{k=1}^{n} \sum_{r=1}^{n} P_{i k}\left(H_{t}^{\top}\left(F_{t} V F_{t}^{\top}\right)^{-1} H_{t}\right)_{k r} P_{r j},
\end{aligned}
$$

of which the compact form is (12). 


\section{References}

1. W. Goffman and V. Newill, "Generalization of epidemic theory," Nature, vol. 204, no. 4955, pp. 225-228, 1964.

2. J. Leskovec, L. A. Adamic and B. A. Huberman, "The dynamics of viral marketing," ACM Transactions on the Web, vol. 1, no. 1, 5, 2007.

3. J. K. Kundan Kandhway, "Optimal control of information epidemics modeled as maki thompson rumors," Communications in Nonlinear Science and Numerical Simulation, vol. 19, no. 12, pp. 4135-4147, 2014.

4. H. S. Rodrigues, "Application of sir epidemiological model: newtrends," International Journal of Applied Mathematics and Informatics, vol.10, pp. 92-97, 2016.

5. C. Chamley, A. Scaglione and L. Li, "Models for the diffusion of beliefs in social networks: An overview," IEEE Singal Processing Magazine, vol. 30, no. 3, pp. 16-29, 2013.

6. W. O. Kermack and A. G. McKendrick, "A contribution to the mathematical theory of epidemics," Proc. Roy. Soc. A, vol. 115, no. 772, pp. 700-721, 1927.

7. F. Liu and M. Buss, "Optimal control for information diffusion over heterogeneous networks," in 55th IEEE Conference on Decision and Control (CDC), pp.141-146, 2016.

8. F. Liu and M. Buss, "Optimal control for heterogeneous node-based information epidemics over social networks," IEEE Transactions on Control of Network Systems, vol. 7, no. 3, pp. 1115-1126, 2020.

9. H. W. Hethcote, "The mathematics of infectious diseases," SIAM Review, vol. 42, no. 4, pp. 599-653, 2000.

10. P. E. Paré, J. Liu, C. L. Beck, B. E. Kirwan and T. Basar, "Analysis, estimation, and validation of discrete-time epidemic processes," IEEE Transactions on Control Systems Technology, vol. 28, no. 1, pp. 79-93, 2018.

11. D. Xue and S. Hirche, "Distributed topology manipulation to control epidemic spreading over networks," IEEE Transactions on Signal Processing, vol. 67, no. 5, pp. 1163-1174, 2019.

12. F. Liu, Z. Zhang and M. Buss, "Robust optimal control of deterministic information epidemics with noisy transition rates," Physica A: Statistical Mechanics and its Applications, vol. 517, pp. 577-587, 2019.

13. R. Garg, M. D. Smith and R. Telang, "Measuring information diffusion in an online community," Journal of Management Information Systems, vol. 28, no. 2, pp. 11-38, 2011.

14. H. Kwakernaak, "Optimal filtering in linear systems with time delays," IEEE Transactions on Automatic Control, vol. 12, no. 2, pp.169-173, 1967.

15. M. Hou and R. J. Patton, "Optimal filtering for systems with unknown inputs," IEEE Transactions on Automatic Control, vol. 43, no. 3, pp. 445-449, 1998.

16. M. V. Basin and J. J. Maldonado, "Optimal controller for uncertain stochastic linear systems with poisson noises," IEEE Transactions on Industrial Informatics, vol.10, no.1, pp. 267-275, 2014.

17. M. Liu, L. Zhang, P. Shi and H. R. Karimi, "Robust control of stochastic systems against bounded disturbances with application to flight control," IEEE Transactions on Industrial Electronics, vol. 61, no. 3, pp. 1504-1515, 2013.

18. M. Liu and P. Shi, "Sensor fault estimation and tolerant control for itô stochastic systems with a descriptor sliding mode approach,” Automatica, vol. 49, no. 5, pp. 1242-1250, 2013.

19. Z. Gao and X. Shi, "Observer-based controller design for stochastic descriptor systems with brownian motions," Automatica, vol. 49, no. 7, pp. 2229-2235, 2013.

20. B. Qu and H. Wang, "SIS epidemic spreading with heterogeneous infection rates," IEEE Transactions on Network Science and Engineering, vol. 4, no. 3, pp. 177-186, 2017.

21. A. H. Jazwinski, Stochastic processes and filtering theory. Courier Corporation, 2007.

22. H. R. Joshi, "Optimal control of an HIV immunology model," Optimal Control Applications and Methods, vol. 23, pp. 199-213, 2002.

23. K. Kandhway and J. Kuri, "How to run a campaign: Optimal control of sis and sir information epidemics," Applied Mathematics and Computation, vol. 231, pp.79-92, 2014.

24. M. Basin, D. Calderon-Alvarez and M. Skliar, "Optimal filtering for incompletely measured polynomial states over linear observations," International Journal of Adaptive Control and Signal Processing, vol. 22, no. 5, pp. 482-494, 2008.

25. G. Bishop, G. Welch, et al., "An introduction to the Kalman filter," Proc. of SIGGRAPH, Course, vol. 8, no. 27599-23175, p. 41, 2001.

26. K. Reif, S. Gunther, E. Yaz and R. Unbehauen, "Stochastic stability of the continuous-time extended Kalman filter," IEE Proceedings-Control Theory and Applications, vol.147, no.1, pp. 45-52, 2000.

27. S. Sarkka, "On unscented Kalman filtering for state estimation of continuous-time nonlinear systems," IEEE Transactions on Automatic Control, vol. 52, no. 9, pp. 1631-1641, 2007.

28. M. V. Basin and P. C. Rodríguez-Ramírez, "Sliding mode controller design for stochastic polynomial systems with unmeasured states," IEEE Transactions on Industrial Electronics, vol. 61, no. 1, pp. 387-396, 2013.

29. M. Basin, New trends in optimal filtering and control for polynomial and time-delay systems. Springer, 2008.

30. J. S. Coleman, Introduction to mathematical sociology. London Free Press, Glencoe, 1964. 


\section{Bionotes}

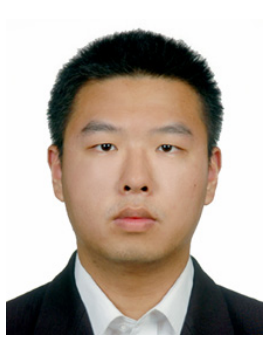

\section{Fangzhou Liu}

Chair of Automatic Control Engineering (LSR), Department of Electrical and Computer Engineering, Technical University of Munich, Theresienstr. 90, 80333, Munich, Germany

fangzhou.liu@tum.de

Fangzhou Liu received the M.Sc. degree in control theory and engineering from Harbin Institute of Technology, Harbin, China, in 2014 and the Doktor-Ingenieur degree in electrical engineering from Technical University of Munich, Germany, in 2019. He is now a Lecturer and Research Fellow with the Chair of Automatic Control Engineering, Technical University of Munich, Germany. He has received the Dimitris N. Chorafas Prize and the Promotionspreis der Fakultät für Elektrotechnik und Informationstechnik for his PhD thesis. His current research interests include networked control systems; modeling, analysis, and control on social networks; and their applications.

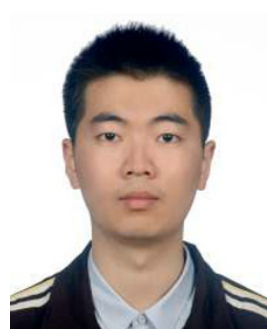

\section{Zengjie Zhang}

Chair of Automatic Control Engineering (LSR), Department of Electrical and Computer Engineering, Technical University of Munich, Theresienstr. 90, 80333, Munich, Germany zengjie.zhang@tum.de

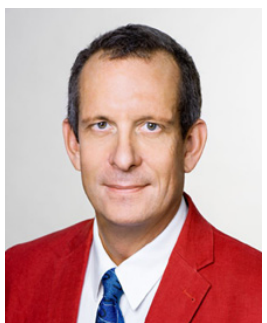

\section{Martin Buss}

Chair of Automatic Control Engineering (LSR), Department of Electrical and Computer Engineering, Technical University of Munich, Theresienstr. 90, 80333, Munich, Germany

mb@tum.de

Martin Buss received the Diploma Engineering degree in electrical engineering from the Technische Universität Darmstadt, Darmstadt, Germany, in 1990 and the Doctor of Engineering degree from the University of Tokyo, Tokyo, Japan, in 1994, both in electrical engineering.

In 1988, he was a Research Student for one year with Science University of Tokyo. From 1994 to 1995, he was a Postdoctoral Researcher in the Department of Systems Engineering, Australian National University, Canberra, ACT, Australia. From 1995 to 2000, he was a Senior Research Assistant and Lecturer in the Chair of Automatic Control Engineering, Department of Electrical Engineering and Information Technology, Technical University of Munich, Munich, Germany. From 2000 to 2003, he was a Full Professor, the Head of the Control Systems Group, and the Deputy Director of the Institute of Energy and Automation Technology, Faculty IV, Electrical Engineering and Computer Science, Technical University Berlin, Berlin, Germany. Since 2003, he has been a Full Professor (Chair) in the Chair of Automatic Control Engineering, Faculty of Electrical Engineering and Information Technology, Technical University of Munich, where he has been in the Medical Faculty since 2008. He is a Fellow of IEEE since 2014. He has been awarded the ERC Advanced Grant SHRINE. His research interests include automatic control, mechatronics, multimodal human system interfaces, optimization, nonlinear, and hybrid discrete-continuous systems.

Zengjie Zhang received his Bachelor and Master degrees from Harbin Institute of Technology, China, in 2013 and 2015 respectively. He is currently a Research Associate at the Chair of Automatic Control Engineering of the Technical University of Munich, Germany, while pursuing the doctoral degree. His research interests include sliding mode control, fault detection and isolation and human-robot collaboration. 